Rov. Chil. Podiatr. 62 (4); 247-251, 1991

\title{
Ventilación mecánica de recién nacidos en Punta Arenas
}

\author{
Julio Montt V. ${ }^{1}$ \\ Mechanical ventilation results at \\ a geographically isolated neonatal unit
}

\begin{abstract}
A retrospective evaluation of 136 newborns treated with mechanical ventilation between 1983 and 1989 at the chilean's shouthernmost regional hospital of Punta Arenas is presented. Seventy five percent of neonates had birthweights under $2,500 \mathrm{~g}$ and $39 \%$ of them were under $1,500 \mathrm{~g}$. Lethality was higher in infants less than $1,500 \mathrm{~g}$ birthweight $(43.4 \%)$. The rost frequent cause of death was periventricular and or intraventricular hemorrhage, occurring in 20 among 26 death5. Leathality was lower among patients with birthweights $1,500 \mathrm{~g}$ throughout $2.499 \mathrm{~g}(16.7 \%)$. The most frequent disease requiring mechanical ventilation was hyaline membrane disease $(62.5 \%)$ : lethality for these patients was $25.9 \%$. Complications developed in $43.4 \%$ of mechanically ven. tila ted newborns, the most frequents being obstruction of the endotracheal tube and athelectasies.

(Key words: mechanical ventilation, newborns, mortality, complications.)
\end{abstract}

1. Servicio de Pedintría, Hospital Regional de Punta Arenas.
En los áltimos años se ha obtenido un descenso importante de la mortalidad peri y neonatal, 
tanto a nivel nacional como regional ${ }^{1}$. Iin rol destacado en estos logros lo ha cumplido la ejecución, a partir de 1980 , de un programa nacional de equipamiento neonatal ${ }^{2}$, que ha favorecido en forma especial a los recién nacidos pretérmino y a aquellos afectados de insuficiencia respiratoria de algún tipo. Entre cstas medidas destacan la introducción de técnicas ventjlatorias tales como presión positiva continua de via aérea y ventilación intermitente a presión positiva.

En la Región de Magallanes este programa nacional se estableció a partir de 1983, con la puesta en marcha de una unidad de neonatología con capacidad de ventilar recién nacidos que constituye un centro de referencia para los otros hospitales de la región: Puerto Natales, Porvenir, Hospital Naval y una clínica privada. Previa a la introducción de este programa, la mortalidad neonatal en clla alcanzaba a 13,7 por 1000 nacidos vivos (NV) el affo 1981 y a 11,7 por $1000 \mathrm{NV}$ el año $1982^{\prime}$. Las tasas especificas de mortalidad por peso de nacimiento variaban entre 500 y 750 por $1000 \mathrm{NV}$ para los pesos entre 1000 y $1.499 \mathrm{~g}$ y entre 181 y 210 por $1000 \mathrm{NV}$, para los pesos entre $1500 \mathrm{y}$ $1999 \mathrm{~g}$ en los años 1981 y $1982^{3}$.

E1 objetivo del presente trabajo es mostrar los resultados y complicaciones del empleo de técnicas de ventilación mecánica en recién nacidos en un hospital regional.

\section{Material y Método}

Se revisaron los registros clínicos de 136 recién nacidos que fueron sometidos a ventibción mecánica en la unidad de neonatología del Hospital Regional de Punta Arenas entre los gños 1983 y 1989. Se consideraron los pesas de nacimiento, causs determinantes de ventila* ción mecánica, complicaciones de ésta, y letalidad global por peso y por enfermedad. Las causas de muerte fueron confirmadas mediante examen de anatomia patológica en 36 de 45 pacientes fallecidos; en los 9 restantes la causa de muerte se identificó clínicamente. Sólo a partir del año 1988 se decidió iniciar la terapia ventilatoria en menores de $1000 \mathrm{~g}$, una vez que se contó con personal estable en la unidad, tanto médico como paramédico. Se utilizaron en el tratamiento ventilatorjo respiradores Bourns BP-200 y Sechrist IV-100. Los criterios de indicación de conexión a ventilación mecánica fueron los clásicos descritos por Reynolds ${ }^{4}$ y Brady y Gregory ${ }^{5}$ : apneas recurrentes e insuficiencia respiratoria, definiendo como hipoxemin valores de $\mathrm{PaO}_{2}$ inferiores a $50 \mathrm{mmHg}$ y oomo hipercapnia valores de $\mathrm{PaCO}_{2}$ superiores a $65 \mathrm{mmHg}$.

Los criterios diagnósticos de algunas de las afecciones mis graves fueron los siguientes: asfixia peri- natal severa se definió como la fresencia de hipoxemia. hipcrapnia $y$ acidosis metabólica, en un recién nacido con antecedentes de cuenta de Apgar igual o inferior a 3 al primer minuto y sin Jecupetación total al quinto minuto, con Apgar igual o inferior a 7 ; hipertensión puimonar primaria se definió como k existencia de derecha izquierda, sin cortocircuito de enfermedad pulmonar o cardíaca; hemorragia periventricular, ir:traventricular o ambas, se diagnosticaron mediante ecografía encefálica, necropsia o punción ventricular postmortem, utilizándose la clasificación de Papille et $a l^{6}$.

\section{Resultados}

Los pesos al nacer de los 136 recién nacidos objetos del estudio fluctuaron entre 600 y 4960 $\mathrm{g} ; 102$ (75\%) pesaron menos de $2.500 \mathrm{~g}$ y 53 (39\%) menos de $1500 \mathrm{~g}$. Las edades gesta. cionales variaron entre 26 y 41 semanas. La duración de la ventilación mecánica fluctuó entre 12 horas y 25 días, con un promedio de 4,5 dís. La mortalidad global fue de $33,1 \%$ (falle. cieron 45 de 136 neonatos ventilados).

La mortalidad fue mayor en el grupo de peso de 1000 a $1499 \mathrm{~g}$, con 22 de 45 pacientes fallecidos $(48,9 \%)$. El grupo de peso de menores de $999 \mathrm{~g}$ sólo representa a un total de 8 pacientes, 4 de los cuales fallecieron, pero que comenzaron a ser ventilados sólo desde el año 1988. La mortalidad global para el grupo de recién nacidos ventilados menores de $1500 \mathrm{~g}$ fue $43,4 \%$, con 26 de 53 pacientes que murieron.

La menor mortalidad correspondió a los grupos de peso de 1500 a $1999 \mathrm{~g}$ y de 2000 a $2499 \mathrm{~g}$, ambos con 16,7\%. En el primer grupo mencionado fallecieron 5 de 30 pacientes tra. tados, $y$ en el segundo grupo murieron 3 de 15 neonatos ventilados. La mortalidad para el grupo de peso de mayores de $2500 \mathrm{~g}$ fue de $31,4 \%$, con 11 de 35 recién racidos fallecidos.

La distribución de los diagnósticos determinantes de ventilación mecánica en los 136 neonatos tratados se observa en la tabla 1. La afección más frecuente fue sindrome de dificultad respiratoria idiopática (SDRI), con 85 casos $(62,5 \%)$. El segundo lugar lo ocupó asfixia perinatal severa con 17 pacientes ventilados $(12,5 \%)$, y el tercero, hernia diafragmática, con 9 recién nacidos tratados $(6,6 \%)$.

En la misma tabla 1 se observan las mortalidades por diagnósticos. Para los neonatos con SDRI, la mortalidad fue $25,9 \%$, con 22 de 85 pacientes tratados que fallecieron. En los casos 
Tabla 1

Distribución y mortalidad según diagnósticos determina ntes de ventilación mecánjea en 136 recién nacidos

\begin{tabular}{|c|c|c|c|c|}
\hline \multirow{2}{*}{ Enfermedad } & \multicolumn{2}{|c|}{ Pacientes } & \multicolumn{2}{|c|}{ Fallecidos } \\
\hline & (n) & $(\%)$ & (n) & $(\%)$ \\
\hline \multicolumn{5}{|l|}{ Síndrome de dificultad respiratoria } \\
\hline idiopática & 85 & 62,5 & 22 & 25,9 \\
\hline Asfixia perinatal severa & 17 & 125 & 6 & 35,3 \\
\hline Hernia diafragmática & 9 & 6,6 & 5 & 55,6 \\
\hline Aspiración de meconio & 6 & 4,4 & 2 & 33,3 \\
\hline Hiper tensión pulmonar primaria & 6 & 4,4 & 4 & 66,7 \\
\hline Bronconeumonía connatal & 5 & 3,7 & 3 & 60,0 \\
\hline Apneas del prematuro & 4 & 29 & 1 & 25,0 \\
\hline Meningitis bacteriana & 2 & 1,5 & 1 & 50,0 \\
\hline Aspiración de leche & $\overline{1}$ & 0,7 & 0 & 0,0 \\
\hline Aspiración de sangre & 1 & 0,7 & 1 & 100,0 \\
\hline Total & 136 & 100,0 & 45 & \\
\hline
\end{tabular}

de asfixia perinatal severa que fueron ventilados murieron 6 de 17 pacientes tratados. De Ios 6 neonatos con diagnóstico de hipertensión pulmonar primaria fallecieron 4.

Se ventilaron 9 recién nacidos con hernia diafragmática congénita ( 8 izquierda y 1 derecha), 5 de los cuales murieron. Todos estos pacientes fueron diagnosticados antes de las primeras 24 horas de vida e intervenidos quirúrgicamente de inmediato, una vez confirmado el diagnóstico; 3 de los 9 neonatos se conectaron a respirador antes de la operación y los 6 restantes después de ésta. Los 5 recién nacidos fallecidos cursaron con cuadro clínico de hipertensión pulmonar irreversible, causa determinante de las muertes.

Se presentaron complicaciones en 59 de 136 pacientes $(43,4 \%)$, las que se enumeran en la tabla 2. La complicación más frecuente fue obstrucción del tubo endotraqueal, con $24,3 \%$ de los casos. El segundo lugar lo ocupó atelectasias, con una incidencia de $22,8 \%$. Neumotórax se presentó sólo en 5,2\% (7 casos). Displasia broncopulmonar $y$ retinopatía del prematuro se presentaron sólo en 2 neonatos cada una. No hubo casos de enterocolitis necrotizante en la muestra estudiada.

Las causas de muerte se analizan en dos grandes grupos de peso: menoreso iguales de $1499 \mathrm{~g}$ y mayores o iguales de $1500 \mathrm{~g}$. La principal causa de muerte en el primer grupo mencionado fue hemorragia periintraventricular, 20 fallecidos
Tabla 2

Tipo y frecuencia de complicaciones presentadas en 136 recién nacidos sometidos a ventilación mecánica

\begin{tabular}{lrr}
\hline $\begin{array}{l}\text { Tipo de } \\
\text { complicación }\end{array}$ & $\mathbf{n}$ & $\%$ \\
\hline Obstrucción tubo & & \\
$\quad$ endotraqueal & 33 & 24,3 \\
Atelectasias & 22 & 22,8 \\
Ductus persistente & 16 & 11,8 \\
Extubación accidental & 15 & 11,0 \\
Neumotórax & 7 & 5,2 \\
Bronconeumonía intra hospitalatia & 6 & 4,4 \\
Septicemia intrahospitalaria & 6 & 4,4 \\
Displasia broncopulmonar & 2 & 1,5 \\
Retinopatía del prematuro & 2 & 1,5 \\
& & \\
\hline
\end{tabular}

de 26 pacientes que la presentaron. La segunda causa de deceso fue bronconeumonia intrahospitalaria, con 3 casos. Las otras causales fueron: neumotórax bilateral, obstrucción del tubo endotraqueal y displasia broncopulmonar, con 1 caso cada una.

En el grupo de peso mayor o igual a $1500 \mathrm{~g}$. la principal determinante de muerte fue hipertensión pulmonar con 9 casos: 5 secundarias a hernia diafragmática y 4 a hipertensión pulmonar primaria. Por hemorragia periintraventricular fallecieron 3 neonatos, al igual que por bronconeumonia connatal. Las otras causas 
de muerte fueron aspiración de meconio, 2 casos, $y$ aspiración de sangre $y$ meningitis bacte. riana, un caso cada una de ellas.

\section{Comentario}

Los recién nacidos menores de $1500 \mathrm{~g}$ presentaron la mayor mortalidad en comparación a los otros grupos de peso que fueron sometidos a ventilación mecánica, lo que se explica por la inmadurez extrema de estos pacientes, que dificultan el tratamiento ventilatorio, en especial la atención de enfemnería relacionada. Además, esta inmadurez determina mayor riesgo de hemorragia periintraventricular, de hecho la principal causa de muerte en este grupo de peso. Los resultados de mortalidad para niños de peso menor de $1500 \mathrm{~g}(43,4 \%)$ no son comparables con los que se mencionan en la literatura extranjera ${ }^{7-13}$, que corresponden a otra realidad en cuanto a organización y recursos de atención intensiva neonata]; sin embargo, estos resultados se comparan favorablemente con las pocas experiencias nacionales publicadas ${ }^{14}, \mathrm{ts}$. No se realizó un análisis separado del grupo de peso de menores de $999 \mathrm{~g}$, por constituir una muestra escasa.

La menor mortalidad que correspondí a los niños de 1500 a $1999 \mathrm{~g}$ y de 2000 a $2499 \mathrm{~g}$ se explica por el mejor peso de nacimiento y la mayor edad gestacional de estos neonatos, con el consecuente menor riesgo de hemorragia periintraventricular extensa (grado 3-4), principal causa de muerte en los de menor peso. Además, la mayor parte de estos pacientes fue tratada por presentar SDRI, entidad que determinó terapias ventilatorias bien normadas y de corta duración, inferiores a 5 dras en promedio, lo que hizo menos complejo el manejo ventilatorio de estos recién nacidos.

E1 grupo de peso mayores de $2500 \mathrm{~g}$ presen. tó la segunda mayor mortalidad del estudio $(31,4 \%)$, lo que se explica porque en este tramo se concentraron afecciones complejas de muy difícil manejo terapéutico y en las cuales se presentaron las mayores mortalidades: hipertensión pulmonar primaria y hernia diafragmática.

E1 análisis según la enfermedad determinante de ventilación mecánica revela que la más fre. cuente fue el síndrome de dificultad respiratoria idiopática. En este grupo, además, se encontró la más baja mortalidad $(25,9 \%)$, lo que se explica por la recuperabilidad de la afección y una dura. ción promedio de la terapia ventilatoria inferior a 5 días; estos resultados son comparables con las publicaciones, tanto nacionales ${ }^{14-16}$ como las extranjeras ${ }^{7-9,11-13,17}$. Las mayores mortalidades por diagnóstico correspondieron a hipertensión pulmonar primaria y a hernia diafragmá. tica, lo que se explica por las grandes dificultades técricas para poder ventilar exitosamente a estos pacientes, la mayor parte de los cuales presentan grados severos de hipertensión pulmonar, y que no siempre responden a terapias agresivas, tales como hiperventilación, parálisís muscular y alcalinización exógena ${ }^{18}$.

$\mathrm{La}$ incidencia de complicaciones fue elevada $(43,4 \%)$, en relación a experiencias internacionales ${ }^{7,8}, 11-13,17,19$, pero similar a lo publicado en la literatura naciona $1^{14,15,20}$. Las complicaciones más frecuentes fueron las propias del manejo del tubo endotraqueal, con obstrucción de éste y atelectasias. Cabe mencionar la baja incidencia de neumotórax $(5,2 \%)$, resultado favorable que explica por el uso de técnicas ventilatorias conservadoras, con tiempos inspiratorios cortos (menores de 0,5 segundos), y a la reducción precoz de las presiones inspiratorias máximas ${ }^{21}$. Destaca tambiến la baja incjdencia de displasia broncopulmonar y de retinopatía del prematuro, con sólo 2 casos cada uno, lo que podría explicarse por ventilaciones de corta duración, y en especial por el bajo número de pacientes con pesos inferiores a 1000 gramos.

Los resultados del presente estudio de ventilación mecánica en un hospital regional permiten afirmar que la introducción de técnicas ventilatorias fue justificada, pese a las limitaciones de recursos que enfrentan los centros hospitalarios públicos. Los desafíos a futuro quedan claramente establecidos, en orden a disminuir la incidencia de complicaciones, sobre todo aquellas dependientes de un mejor manejo de la vía aérea artificial. Además, el otro gran desafío es mejorar los resultados de letalidad del grupo de peso de 1000 a $1499 \mathrm{~g}$, paso previo a mejorar los resultados terapéuticos en neonatos menores de $999 \mathrm{~g}$.

\section{Resumen}

Se revisaron las fichas clínicas de 136 recién nacidos tratados con ventilación mecánica entre 1983 y 1989 en el Hospital Regional de Punta 
Arenas. En 75\% de ellos el peso de nacimiento era inferior a $2500 \mathrm{~g} \mathrm{y} 39 \%$ pesaban menos de $1.500 \mathrm{~g}$ al nacer. La mayor letalidad se registró en los niños cuyos pesos eran $\leqslant 1499 \mathrm{~g}$ $(43,4 \%)$, siendo la causa de muerte más frecuente hemorragia periventricular o intraventricular (20 de 26 neonatos). Las letalidades más bajas se observaron entre los recién nacidos con pesos al racer de 1500 a $2499 \mathrm{~g}(16,7 \%)$. La enfermedad más frecuente como causa de indicación de apoyo mecánico de la ventilación fue el sindrome de dificultad respiratoria idiopática $(62,5 \%)$, cuya letalidad en estos casos fue de $25,9 \%$. Se observaron complicaciones en $43,4 \%$ de los pacientes, siendo las más frecuentes obstrucción del tubo endotraqueal y ate. lectasis.

(Palabras claves: recién nacidos, apoyo ventjlatorio, ventilación mecánica, letalidad, complicaciones, hospitales regionales.)

\section{Referencias}

1. Ministerio de Salud, Chile: Indicadores Biodemográficos. Chile, 1989.

2. Ferreiro, M.; Beca, J.P.; Maler, R.; Vaisman, S.: [mplementación en la atención neonatal en Chile. Pediatría (Santiago), 1976; 19: 282.

3. Servicio de Salud Magalianes, Chile: Informe sobre mortalidad peri y neonatal, años 1980 1989. Chile, 1990.

4. Reynolds, $O_{\text {: }}$ Ventilator therapy. In: Thibeault, D.W. and Gregory, G.A.: Neonatal Pulmonary Care. Calıfornia. Addison-Wesley Publishing Company, 1979: 217-236.

5. Brady, J,P y Gregory, G.A.: Ventilación asistida, En: Klaus, M.H. y Fanaroff, A.A. Asistencia del recién nacido de alto riesgo. Buenos Aires. Editorial Panamericana, $1981 ; 215-232$

6. Papille, LA,; Burstein, J.; Burstein, R, Koffler, $H .:$ incidence and evolution of subependimal and intraventricular hemorrhage: A study of infants with birthweights less than 1.500 g. J Pediatr $1978 ; 92 * 529-534$.

7. Schreiner, R.L, Kisling, J.A.; Evans, G.M. et al.: Improved survival of ventilated neonates with modern intensive care. Pediatrics 1980; 66: 985 987.
8. Cohen, R.S.; Stevenson, D.K.; Molachowski, $N$. et al.: Favorable results of neonatal intensive care for very low birthweight infants. Pediatrics 1982; 69: $621-625$.

9. Saigal, $\boldsymbol{S}$., Rosenbaum, P.; Stoskopf, $\boldsymbol{B}$. et at.: Follow-up of infants 501-1 500 grams birthweight delivered to residents of a geographically defined segion with perinatal intensive care facilities. J Pediatr 1982;100: 606-613

10. Raju, T: An Epidemiologic study of Very and Very Very Low Birth Weight Infants. Clin Perinatol 1986; 13: 233-250.

11. Marlow, N.; Chiswick, M.L.: Neurodevelopmental outcome of babies weighing less than 2.001 grams at birth: influence of Perinatal transfer and Mechanical Ventilation. Arch Dis Child 1988; 63: 1069-1074.

12. Doyle, L.W.; Murton, L.J.; Kitchen, W.H.: Mortality with increasing assisted ventilation of very low birthweight infants. Am J Dis Child 1989; 143: 223-227.

13. Powers, W.F.; Hegwood, P.D.: Survival and ventilatory course of a regional cohort of Very Low Birthweight infants. Am J Periratol 1989; 6: 427-432.

14. Rodríguez, M.S.; Bancalari, A.; Pandolfi, E.: Ventilación Mecánica en Unidad de Neonatología. Rev Chil Pediatr 1986;57: 350-354.

15. Contreves, J.: Experjencia en Ventilación Mecánica en el Recién Nacjiło. Bol Hosp Viña del Mar 1986; 42: 227-230.

16. Weldt, E., Alarcón, J.; Vaisman, S.: Morbilidad y Mortalidad de Recién Nacidos con peso de nacimiento inferior a 1,500 gramos. Rev Chil Podiats 1984; 55: 327-330.

17. Bennett, F.C.; Robinson, N.M.; Sells, C.S.: Hyaline membrane disease, bir thweight and gestational age. An J Dis Child 1982: 136: 888-891.

18. Duara, S.; Gewitz, M.H. Fox, W.W.: Use of me chanical ventilation for persistent pulmonary hypertension of the netwborn. Clin Perinatol 1984; 11: 641-652.

19. Stavis, R.L.; Krouss, A.N.: Complications of neonatal intensive care. Clin Perinatol 1980; $7: 107-124$.

20. Cerda, M.: Soovedra, O.; Aspillaga, M.; Mesa, T. et al.: Ventikción Mecánica en insuficiencia respiratoria de origen pulmonar. Rev Chil Pediatr 1984:55: 25-28.

21. Thibeaul, D.W.: Pulmonary barotrauma, In: Thibeault, D.W. and Gregory, G.A.: Neonatal Pulmonary Care. California, Addison-Wesley Publishing Company, 1979: 307-316. 